\title{
Hypercoagulable State Induced Spinal Cord Stroke After Coronavirus Disease 19 Infection
}

\author{
Lisda Amalia \\ Department of Neurology, Faculty \\ Medicine, Universitas Padjadjaran/RSUP \\ Dr. Hasan Sadikin Bandung, Bandung, \\ Indonesia
}

Background: Spinal cord stroke after coronavirus disease 19 (COVID-19) infection is rare, and limited cases have been reported. Spinal cord ischemia after COVID-19 infection is related to increased coagulopathy with thromboembolic consequences. Patients with COVID-19 may have a hypercoagulable state and an increased rate of thromboembolic events, such as occlusion in the spinal artery.

Case Presentation: We report a male case with confirmed COVID-19 infection, aged 60 years, with flaccid paraplegia, hyporeflexia, loss of sensation below the 12th thoracic level, loss of autonomic function, bilateral positive Babinski sign 14 days after the onset of flu-like symptoms, and elevated serum D-dimer and fibrinogen levels. There was stenosis of the spinal artery at the 12th thoracic level in magnetic resonance imaging and magnetic resonance angiography. $\mathrm{He}$ showed improvement in motor strength of the lower limb (walking with assistance), numbness and pain, and urine and fecal retention after receiving a subcutaneous anticoagulant.

Conclusion: COVID-19 can damage endothelial cells and activate thrombotic pathways, which can lead to clinical thromboembolic complications, such as occlusion in the spinal artery, resulting in spinal cord stroke.

Keywords: COVID-19 infection, hypercoagulable state, spinal cord stroke

\section{Introduction}

In late December 2019, reports of a severe acute respiratory illness emerged from Wuhan (China), which was established as coronavirus disease 2019 (COVID-19); it involved a severe acute respiratory syndrome and rapidly spread to other regions. ${ }^{1}$ The World Health Organization (WHO) declared COVID-19 as a pandemic on March 11, 2020, because of the rapid global spread of the disease, and on October 14, 2021, over 239 million confirmed cases of COVID-19 were reported globally, which included over 4.8 million deaths. ${ }^{2}$ The first two cases of COVID-19 in Indonesia were reported on March 2, 2020. By January 12, 2021, there were 846,765 confirmed COVID-19 cases and 24,645 deaths in Indonesia. ${ }^{3}$

Interestingly, several reports of COVID-19 cases indicated neurological symptoms of both the central (CNS) and peripheral nervous systems, and the most common serious CNS complications are cerebrovascular diseases. ${ }^{4}$ Spinal cord stroke after COVID-19 infection is rare, and limited cases have been reported. ${ }^{5-7}$

We discuss a case report of spinal cord stroke following acute COVID-19 pneumonia caused by anterior spinal artery occlusion. Written informed consent to publish case details and any accompanying images was provided by the patient. Dr. Hasan Sadikin General Hospital Bandung Human Research Ethics Committee approved this consent process.
Correspondence: Lisda Amalia Department of Neurology, Faculty Medicine, Universitas Padjadjaran/RSUP Dr. Hasan Sadikin Bandung, Jl. Pasteur 38, Bandung, 40I6I, Indonesia

Email dr.lisda@gmail.com 


\section{Case Presentation}

A 60 -year-old male was admitted to the emergency unit with weakness and numbness in both lower limbs with girdle-like pain at the lower thoracic level after 14 days of fever and upper respiratory tract infection. Two days later, symptoms progressed to complete lower-limb paralysis, with loss of sensation below the 12th thoracic level. He also had urine and fecal retention. Coronary artery disease as a comorbidity was recorded 10 months earlier, and the patient underwent a primary coronary intervention procedure because of three-vessel coronary artery occlusion. He had hypertension, which was regularly controlled, and had ceased smoking for 20 years. Routine medication before admission was $81 \mathrm{mg}$ aspirin once daily, $2.5 \mathrm{mg}$ bisoprolol once daily, and $20 \mathrm{mg}$ atorvastatin once daily.

Upon examination, the patient was fully alert, and the cranial nerves were unaffected. Both lower limbs had complete paralysis, with hypotonia, hyporeflexia, and a positive Babinski sign bilaterally. Sensation to light touch diminished below the 12th thoracic level, with loss of pricking pain, temperature sensation, and vibration (tested by a tuning fork).

Laboratory tests were positive for severe acute respiratory syndrome-coronavirus-type 2 (SARS-CoV-2) based on a polymerase chain reaction test using a nasopharyngeal swab. Serum D-dimer $(4.06 \mathrm{mg} / \mathrm{L})$, fibrinogen $(663 \mathrm{mg} / \mathrm{dL})$ (normal values: D-dimer up to $0.55 \mathrm{mg} / \mathrm{L}$ and fibrinogen 238 $498 \mathrm{mg} / \mathrm{dL}$ ), and erythrocyte sedimentation rate (ESR) in the first hour ( $98 \mathrm{~mm} /$ hour; reference ESR value $<15 \mathrm{~mm} / \mathrm{h}$ ) were elevated. White blood cell (WBC) count $7.9 \times 10^{9} / \mu \mathrm{L}$, red blood cell (RBC) count $4.18 \times 10^{6} / \mu \mathrm{L}$, hemoglobin $11.70 \mathrm{~g} /$ $\mathrm{dL}$, platelet count $188 \times 10^{3} / \mu \mathrm{L}$, neutrophils $80.9 \%$ (the neutrophil count was high), lymphocytes $15 \%$ (low), eosinophils 0.70 (low), basophils $0 \%$, and monocytes $2 \%$ (normal values: WBCs $4-10 \times 10^{3} / \mu \mathrm{L}$, hemoglobin $12-15 \mathrm{~g} / \mathrm{dL}$, platelet count $140-450 \times 10^{3} / \mu \mathrm{L}$, neutrophils $40 \%-75 \%$, lymphocytes $20 \%-45 \%$, monocytes $2 \%-10 \%$, eosinophils $2-6 \%$, and basophils $0-1 \%$ ). All electrolytes were within the normal limit. Liver and renal functions were also within the normal limit. A sagittal T2-weighted magnetic resonance imaging (MRI)magnetic resonance angiography image of the thoracic and lumbar spine (Figure 1A and B) showed a drop in signal of the anterior spinal artery at the 12 th thoracic level. He was treated with heparin $5000 \mathrm{IU}$ every 8 hours, $81 \mathrm{mg}$ aspirin once daily, $2.5 \mathrm{mg}$ bisoprolol once daily, $20 \mathrm{mg}$ atorvastatin once daily, $5 \mathrm{mg}$ lisinopril once daily, $500 \mathrm{mg}$ mecobalamin every 8 hours, and $300 \mathrm{mg}$ gabapentin once daily for neuropathic pain symptoms.
After 44 days of hospitalization, he showed an improvement in lower limb motor strength (walking with assistance) and exhibited numbness, pain, and urine and fecal retention.

\section{Discussion}

COVID-19 infection can involve rapid multiorgan dysfunction and varies in presentation from asymptomatic to severe. ${ }^{1}$ Interestingly, several reports of COVID-19 cases have shown neurological symptoms of both the CNS and peripheral nervous system, ${ }^{8}$ and the most common serious CNS complications are cerebrovascular diseases, which indicate direct and immune-mediated effects of the virus on the nervous system. ${ }^{4}$

Stroke is reported in $2 \%-6 \%$ of hospitalized patients with COVID-19, and acute cerebrovascular disease is the most severe complication of COVID-19 due to abnormalities in cascade coagulation according to cohort studies. ${ }^{1}$ SARS-CoV-2 can induce inflammatory response syndrome, higher production of pro-inflammatory mediators (cytokines and chemokines), activation of thrombotic pathways, and a hypercoagulation state due to abnormalities in cascade coagulation, which contributes to the cerebrovascular event. ${ }^{9}$ These responses may be because the viral invasion of the vascular endothelium (endotheliitis) contributes to vascular ischemia of the spinal cord. ${ }^{9}$ Spinal cord stroke after COVID-19 infection is rare, and limited cases have been reported. ${ }^{5-7}$

Tumor necrosis factor- $\alpha$, interleukin (IL)-6, and IL-1 are important cytokines that inhibit intrinsic anticoagulation pathways in COVID-19 patients. This reaction facilitates coagulation activation and thrombin formation, which induces occlusion in the vascular system. ${ }^{10}$ Furthermore, local apoptosis and potent inflammatory cytokines are induced by inflammation in pericytes and infection in endothelial cells, and intravascular coagulopathy is activated following the inflammatory process in the pulmonary alveoli. Patients with COVID-19 may have a hypercoagulable state and an increased rate of thromboembolic events, such as occlusion in the spinal artery. ${ }^{10}$

In this case report, we presented a typical ischemic pattern of spinal cord stroke after COVID-19 infection, with an acute onset, a complete neurological problem at the 12th thoracic level, and a hypercoagulable state confirmed by elevated D-dimer levels and hyperfibrinogenemia. Intravascular thrombosis pathways leading to microvascular and macrovascular thrombosis complications occur after intravascular thrombosis pathways are activated because of systemic cytokine production. Cytokines and platelets interact with neutrophils, which 


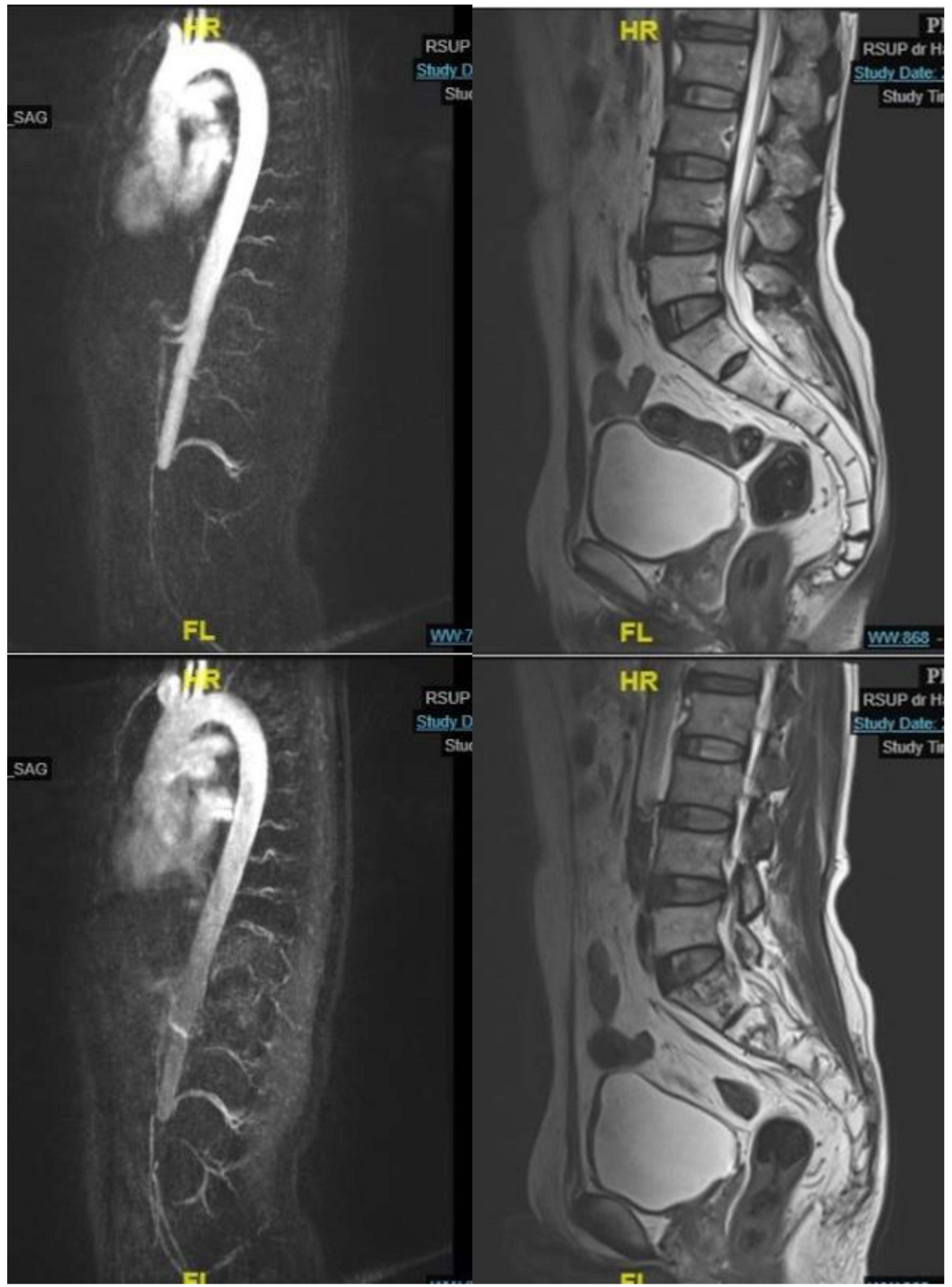

A

B

Figure I Magnetic resonance imaging (MRI). (A) Magnetic resonance angiography showing a drop in signal at the I2th thoracic level. (B) MRI showing no ischemic or demyelinating lesion. 
stimulate thrombin production and fibrin deposition. Excess fibrin deposition and fibrinolysis shutdown lead to intravascular thrombosis and eventually clinical thromboembolic complications, such as occlusion in the spinal artery, which leads to spinal cord stroke. ${ }^{11}$ However, further studies are needed to explore the causal relationship.

\section{Limitation of the Study}

This case study has several limitations. MRI was not repeated following the administration of the anticoagulant or following neurological deficit recovery.

\section{Conclusion}

Some COVID-19 cases exhibit neurological symptoms, with the most common serious CNS complications being cerebrovascular diseases, such as spinal cord stroke. SARS-CoV-2 can damage endothelial cells and activate inflammatory and thrombotic pathways due to an abnormal coagulation cascade, which eventually result in clinical thromboembolic complications, such as occlusion in the spinal artery, resulting in spinal cord stroke. Complications following COVID-19 infection are increasing and are often unfamiliar to healthcare professionals. Thus, clinicians, especially neurologists, should be informed of such severe complications to enable prompt diagnoses and appropriate treatments to decrease spinal cord stroke-related morbidity and reduce public health burdens.

\section{Funding}

No funding was obtained for this research.

\section{Disclosure}

The author reports no conflicts of interest for this work.

\section{References}

1. Huang C, Wang Y, Li X, et al. Clinical features of patients infected with 2019 novel coronavirus in Wuhan, China. Lancet. 2020;395:497-506. doi:10.1016/S1040-6736(20)30183-5

2. World Health Organization. Rolling updates on coronavirus disease (COVID-19). Geneva: World Health Organization; 2020 [cited January 13, 2021]. Available from: https://www.who.int/emergen cies/diseases/novel-coronavirus-2019/events-as-they-happen.

Accessed December 7, 2021.

3. Ministry of Health Republic of Indonesia. COVID-19 crisis center. Jakarta: Ministry of Health Republic of Indonesia; 2021 [Cited January 12, 2021] Available from: https://www.pusatkrisis.kemkes. go.id. Accessed December 7, 2021.

4. Ellul MA, Benjamin L, Singh B, et al. Neurological associations of COVID-19. Lancet Neurol. 2020;19:767-783. doi:10.1016/s14744422(20)30221-0

5. AlKetbi R, AlNuaimi D, AlMulla $M$, et al. Acute myelitis as a neurological complication of COVID-19: a case report and MRI findings. Radiol Case Rep. 2020;15:1591-1595. doi:10.1016/j. radcr.2020.06.001

6. Munz M, Wessendorf S, Koretsis G, et al. Acute transverse myelitis after COVID-19 pneumonia. J Neurol. 2020;267:2196-2197. doi:10.1007/s00415-020-09934-w

7. Sarma D, Bilello LA A case report of acute transverse myelitis following novel coronavirus infection. Clin Pract Cases Emerg Med. 2020;4:321-323. doi: 10.5811/cpcem.2020.5.47937.

8. Sotoca J, Rodríguez-álvarez Y. COVID-19-associated acute necrotizing myelitis. Neurol Neuroimmunol Neuroinflamm. 2020;7:e803. doi:10.1212/nxi.0000000000000803

9. Reddy ST, Garg T, Shah C, et al. Cerebrovascular disease in patients with COVID-19: a review of the literature and case series. Case Rep Neurol. 2020;12:199-209. doi:10.1159/000508958

10. Getu S, Tiruneh T, Andualem H, et al. Coagulopathy in SARS-CoV-2 infected patients: implication for the management of COVID 19. J Blood Med. 2021;12:635-643. doi:10.2147/JBM.S304783

11. Ortega-Paz L, Capodano D, Montalescot G, Angiolillo DJ. Coronavirus disease 2019-associated thrombosis and coagulopathy: review of the pathophysiological characteristics and implications for antithrombotic management. $J$ Am Heart Assoc. 2021;10:e019650. doi:10.1161/JAHA.120.019650
Journal of Blood Medicine

\section{Publish your work in this journal}

The Journal of Blood Medicine is an international, peer-reviewed, open access, online journal publishing laboratory, experimental and clinical aspects of all aspect pertaining to blood based medicine including but not limited to: Transfusion Medicine; Blood collection, Donor issues, Transmittable diseases, and Blood banking logistics; Immunohematology; Artificial and alternative blood based

\section{Dovepress}

therapeutics; Hematology; Biotechnology/nanotechnology of blood related medicine; Legal aspects of blood medicine; Historical perspectives. The manuscript management system is completely online and includes a very quick and fair peer-review system. Visit http://www.dovepress.com/testimonials.php to read real quotes from published authors. 